\title{
EL TRIPLE ASCENSO DE PIETRO VELASCO. CAUTIVERIO, PROMOCIÓN DEL TALENTO Y "ASIMILACIÓN ANTAGONISTA" ENTRE CRISTIANDAD E ISLAM (1583-1608)
}

\section{THE TRIPLE RISE OF PIETRO VELASCO. CAPTIVITY, TALENT PROMOTION AND "ANTAGONISTIC ASSIMILATION" BETWEEN CHRISTENDOM AND ISLAM (1583-1608)}

\author{
Valentina Oldrati \\ Universidad Autónoma de Madrid
}

\begin{abstract}
RESUMEN
El objetivo de este ensayo es analizar la promoción del talento como medio de salida desde una condición de cautiverio o esclavitud en el contexto de la llamada rivalidad Habsburgo-otomana. Para ello, se analizará el caso de Pedro Velasco, un ex-cautivo dominicano que tras renegar entró a formar parte del séquito y de la armada del Kapudan Pachá Scipione Cicala, para luego volver al catolicismo y pasar al servicio de Florencia primero, y de España después (1590-1608 ca).
\end{abstract}

A través de este caso, se reflexionará sobre el vínculo que se creó en el siglo XVI entre conversión religiosa y posibilidad de ascenso socio-económico, evidenciando como, en numerosas ocasiones, fueron las grandes rivalidades internacionales y los equilibrios geopolíticos a permitir y orientar las carreras de los (ex) cautivos más audaces y calificado

Palabras clave: Renegados, cautiverio, espionaje, Gran Ducado de Toscana, Monarquía Hispánica, Imperio Otomano.

\begin{abstract}
The arrival of the colonizers on the Caribbean islands was a milestone in history. It influenced the way we perceive the world today. The way the island of San Juan Bautista (Puerto Rico) was colonized helps to understand how the production system was modified, with a large part in the slave work. One of the ways of perceiving it is the analysis of the different archival sources that mention which people were in charge of doing this work. One of the most important families being that of Ponce de León who together with other personalities supported a slavery model, whose footprint, even today,
\end{abstract}

Keywords: Renegades, captivity, espionage, Grand Duchy of Tuscany, Spanish Monarchy, Ottoman Empire. 


\section{RESUM}

\section{EL TRIPLE ASCENS DE PIETRO VELASCO. CAPTIVITAT, PROMOCIÓ DEL TALENT I "ASSIMILACIÓ ANTAGONISTA" ENTRE CRISTIANDAT I ISLAM (1583-1608)}

L'objectiu d'aquest assaig és analitzar la promoció del talent com via de fuita des d'una condició de captiveri o esclavitud en el context de l'anomenada rivalitat Habsburg-otomana. Per aquesta raó, s'analitzarà el cas de Pedro Velasco, un excaptiu dominicà que després de renegar va entrar a formar part del seguici i de l'armada del Kapudan Pachá Scipione Cicala, per a seguidament tornar al catolicisme i passar al servici de Florència primer, i d'Espanya després (ca. 1590-1608). Mitjançant aquest cas, es reflexionà al voltant del vincle que es va crear al segle XVI entre la conversió religiosa i la possibilitat $d^{\prime}$ 'ascens socioeconòmic, evidenciant com, en nombroses ocasions, van ser les grans rivalitats internacionals i els equilibris geopolítics els que van permetre i orientar les carreres dels (ex)captius més audaços i qualificats.

Paraules clau: Renegats, captiveri, espionatge, Gran Ducat de Toscana, Monarquia Hispànica, Imperi Otomà. 


\section{INTRODUCCIÓN}

El objetivo de este ensayo es analizar la promoción del talento como medio de salida desde una condición de esclavitud o desventaja en el contexto de la llamada rivalidad Habsburgo-otomana. Se examinarán así las trayectorias de vida trans-religiosas y trans-imperiales que esta rivalidad involuntariamente generó. ${ }^{1}$

Concretamente, se analizará el caso de Pietro Velasco, un cautivo de origen calabrés que tras renegar entró a formar parte del séquito y de la armada del Kapudan Pasha otomano Cigalazade Yusuf Sinan Pasha, ${ }^{2}$ también renegado, para luego volver al catolicismo y pasar al servicio de Florencia primero, y de España después. A través de los periplos de Velasco se reflexionará sobre el vínculo que se creó en el siglo XVI entre conversión religiosa y posibilidad de ascenso socio-económico, evidenciando como, en numerosas ocasiones, fueron las grandes rivalidades internacionales y los equilibrios geopolíticos a permitir y orientar las carreras de los cautivos y ex-cautivos más audaces y calificados.

En este contexto, nos serviremos del concepto de talento no solamente para indicar aquellas capacidades innatas que una persona tiene para aprender o desarrollar con habilidad una actividad determinada, sino también para señalar todas aquellas competencias que el ser humano puede adquirir a través de la experiencia. En nuestro caso, al margen de las mencionadas (y difícilmente verificables) aptitudes innatas, nos referimos a los conocimien-

1 Debemos a Natalie Rothman la introducción de esta categoría en la historiografía del Mediterráno moderno. ROTHMAN, E. Natalie (2012), Brokering Empire: Trans-Imperial Subjects between Venice and Istanbul, Cornell University Press, Ithaca. 
tos lingüísticos que Pietro Velasco adquirió viviendo entre las dos orillas del Mediterráneo y, más aún, a las competencias de tipo naval y militar que este obtuvo navegando bajo distintos patrones.

\section{Cautiverio, conversiones Y promoción del talento}

El reinado de Felipe II es una etapa crucial para el estudio de las relaciones entre España y el Imperio Otomano. En el Mediterráneo, la expansión otomana posterior a la caída de Constantinopla agudiza enormemente y en el espacio de pocas décadas las necesidades europeas de conocer los movimientos y las intenciones del enemigo. Especialmente involucrada en la guerra contra el Turco, la Monarquía Hispánica es una de las primeras potencias católicas en crear una red de agentes y espías operativo en tierras otomano-berberiscas. ${ }^{3}$

A partir de los años treinta del siglo XVI -época de la conquista española del Peñón de Argel (1529) y culmen de la consolidación otomana en el Norte de África-y, más aún, tras las derrotas de Mostaganem (1558) y de los Gelves (1560) - es decir, tras la llegada a Estambul de un gran número de prisioneros españoles e italianos, y la consecuente puesta en marcha de las operaciones necesarias para su rescate - los Austrias de España empiezan a invertir recursos crecientes en la infiltración de espías en los centros neurálgicos más destacados del Imperio otomano.

Su labor, de lo más heterogénea, varía desde la recogida de informaciones sobre el estado de la armada turca, a la orquestación de sabotajes (in primis, la quema del Arsenal), hasta la instigación de revueltas entre cuer-

2 El título de Kapudan Pasha corresponde grosso modo al de Capitán General de la Mar.

3 La importancia de los servicios de inteligencia en la España moderna ha sido infravalorada durante largo tiempo por la historiografía tradicional, más interesada al estudio de la diplomacia oficial, sobre todo en su vertiente bélica. A partir de los años ochenta, un número cada vez mayor de historiadores - recordamos ante todo a Emilio Sola, Carlos Carnicer García, Javier Marcos Rivas, Diego Navarro Bonilla, Rafael Vargas Hidalgo, María José Rodríguez-Salgado y Enrique García Hernán - ha empezado a romper el silencio, dando por primera vez un rostro a los hombres y al funcionamiento de los servicios de inteligencia de la Monarquía Habsburgo. La relación con el Imperio otomano, clave para la gran mayoría de los autores citados, ha llamado recientemente la atención de una nueva generación de historiadores turcos, que gracias a sus conocimientos lingüísticos han empezado a integrar las fuentes otomanas con las fuentes europeas, abriendo las puertas a unas nuevas y estimulantes fronteras de investigación. Entre ellos, señalamos primeramente a Emrah Safah Gürkan, experto de las relaciones entre Otomanes y Habsburgo en el siglo XVI. Asimismo, aunque más enfocados a las relaciones entre Turquía y Francia, señalamos los trabajos de Güneş Işıksel. 
pos sociales tradicionalmente vistos como inestables, como por ejemplo los jenízaros o los cautivos de rescate. ${ }^{4}$

Junto con la infiltración de agentes en tierras enemigas, la Monarquía hispánica desarrolla también cierta propensión hacia la acogida de aquellos desertores que estén dispuestos a ofrecer información y servicios encaminados a la derrota del Gran Turco. Si bien la eficacia de un buen espía estaba supeditada a su ubicación, siempre es posible sacar provecho de un arráez bien formado, que sepa dónde y cuándo atacar para hacer el mayor daño posible.

Los llamados "renegados" -variado colectivo humano formado por aquellos cristianos que, tras llegar a tierras islámicas, se convierten y reconstruyen sus vidas bajo la Media Luna- terminan siendo una pieza clave en este juego de traiciones dobles y triples. ${ }^{5}$ Especialmente dentro del Imperio otomano, donde es posible una cierta movilidad social de tipo meritocrático, algunos renegados alcanzan un éxito personal y profesional que les lleva a ocupar altos cargos políticos, militares y administrativos. Frecuentemente, se trata de ex-cautivos cristianos nunca rescatados que se han convertido al islam para adquirir la libertad.

4 Referencia absoluta son los estudios de Emilio Sola. En particular: SOLA, Emilio (2005), Los que van y los que vienen: información y fronteras en el Mediterráneo clásico del siglo XVI, Universidad de Alcalá, Alcalá de Henares. Mucho material, incluso fuentes de archivo, ha sido también publicado en el web www.archivodelafrontera.com. Más recientemente, Gennaro Varriale ha publicado su tesis doctoral, un instrumento muy útil para el estudio de los servicios de información hispano, con especial énfasis en el papel de Nápoles: VARRIALE, Gennaro (2014), Arrivano li Turchi: guerra navale e spionaggio nel Mediterraneo (1532-1582), Città del Silenzio, Novi Ligure.

5 Lecturas recomendadas sobre los cristianos renegados en la época moderna: GRAF, Tobias (2017), The Sultan's Renegades: Christian-European Converts to Islam and the Making of the Ottoman Elite, Oxford University Press, Oxford; KRSTIC, Tijana (2011), Contested Conversions to Islam: narratives of Religious Change in the Early Modern Ottoman Empire, Stanford University Press, Stanford; FIUME, Giovanna (2010), Schiavitù mediterranee: corsari, rinnegati e santi di età moderna, Mondadori, Milán; SIEBENHÜNER, Kim (2008), "Conversion, mobility and the Roman Inquisition in Italy Around 1600", Past \& Present, , n 200-1, pp. 5-35; MESSANA, Maria Sofia (2007), "La 'resistenza' musulmana e i 'martiri' dell'islam: moriscos, schiavi e cristiani rinnegati di fronte all'Inquisizione spagnola di Sicilia", Quaderni storici, n 42-126, pp. 743-772; GARCÍA-ARENAL, Mercedes (2001), Conversions islamiques: identités religieuses en islam méditerranéen, Maisonneuve et Larose, París; SCARAFFIA, Lucetta (1993), Rinnegati: per una storia dell'identità occidentale, Laterza, Roma; GONZALEZ-RAYMOND, Anita (1989), La Croix et le Croissant: les inquisiteurs des îles face à l'islam, 1550-1700, Centre national de la recherche scientifique, París; BENNASSAR, Bartolomé (1989), Los cristianos de Alá: la fascinante aventura de los renegados, Nerea, Madrid; y ROSTAGNO, Lucia (1983), Mi faccio turco: esperienze ed immagini dell'islam nell'Italia moderna, Istituto per l'Oriente C.A. Nallino, Roma. 
Las "élites renegadas" llaman la atención de los servicios de inteligencia de prácticamente todos los países cristianos del Mediterráneo, que consideran a los musulmanes nuevos algo más fáciles de reclutar que los moros de nacimiento, por una serie de razones que van desde su supuesta propensión a la traición, ${ }^{6}$ hasta la posibilidad de aprovecharse de los vínculos lingüísticos y familiares que muchos apóstatas conservaban con sus tierras natales. $^{7}$

Para poder entender cómo nuestro discurso quiere destacar el vínculo que une cautiverio, apostasía y promoción del talento, es necesario que abramos un paréntesis. Las órdenes y las cofradías religiosas que en los siglos $\mathrm{XVI}$ y XVII se dedican al rescate de cautivos cristianos, no rescatan a los cristianos de Alá. ${ }^{8}$ En esta óptica, para los musulmanes que comercian con seres humanos la conversión al islam de todo cautivo de rescate supondría un daño económico a evitar a toda costa. No es totalmente falso, pero existen estrategias comerciales alternativas a la venta de los cautivos.

Concretamente, los cautivos pueden ponerse de acuerdo con sus patrones para obtener el permiso para marcharse y recolectar autónomamente el dinero necesario para autorescatarse. Este mecanismo, llamado manumisión, beneficia al corso y a la piratería porque la navegación es una excelente manera para acumular rápidamente la suma necesaria para un rescate. ${ }^{9}$ Paralelamente la manumisión resulta ser una operación ventajosa para los mercaderes de esclavos, ya que no interrumpe las relaciones entre dueños y cautivos, sino que transforma su naturaleza generando un vínculo de patronazgo que establece obligaciones jurídicas y económicas para ambos contratistas.

6 Sobre la cuestión de la propensión a la traición: BUNES, Miguel Angel de (1989), La imagen de los musulmanes y del Norte de África en la España de los siglos XVI y XVII, CSIC, Madrid, pp. 232-236.

7 Sobre el mantenimiento de dichos vínculos: GRAF, Tobias (2014), "Of half-lives and double-lives: 'Renegades' in the Ottoman Empire and Their Pre-Conversion Ties, ca. 1580-1610", en FIRGES, Pascal et al. (eds.), Well-connected domains, Brill, Leiden, pp. 131-149; y KUNT, Metin Ibrahim (1974), "Ethnic-Regional (cins) Solidarity in the Seventeenth-Century Ottoman Establishment", International Journal of Middle East Studies, n 5-3, pp. 233-239.

8 Otra cosa son los rescates privados, que no están vinculados a estatutos de naturaleza religiosa.

9 La piratería es peligrosa y no es un trabajo para todos. Muchos prefieren volver a su tierra natal. Los que vuelven a España y apelan al rey para obtener una limosna correspondiente a la deuda contraída se conocen como cortados. Sin embargo, no todos intentan devolver la suma pactada, y no faltan los ex-cautivos cristianos que cuestionan la legitimidad de un contrato estipulado con un infiel. 
Por añadidura, la manumisión permite hacer la voluntad de Alá: la liberación de un cautivo se acompaña siempre a su conversión porque sólo un musulmán puede acceder ese estado de semi-libertad que le permitirá trabajar y pagar por su rescate. ${ }^{10}$ En este sentido estamos ante una audaz forma de inversión, que apuesta en el paso del tiempo y, sobre todo, en las capacidades de los cautivos antes que en una recompensa inmediata. Los renegados que llegan al islam por este camino aportan fuerza al mundo que les asimila quitando otras tantas al mundo que dejan atrás.

Dando un paso más allá en nuestro razonamiento, este mismo discurso puede aplicarse también a las numerosas negociaciones con renegados -eso sí, de alto rango- ${ }^{11}$ que los servicios de inteligencia de la Monarquía hispánica llevan a cabo entre la segunda mitad del siglo XVI y las primeras décadas de la centuria siguiente. Asimismo, y al margen de toda cuestión religiosa, las rivalidades entre potencias cristianas duplicarán estas estrategias de asimilación también en suelo europeo.

Gracias a los periplos de Pietro Velasco veremos entrar en acción a todos estos mecanismos, y reflexionaremos sobre las turbulentas relaciones que los Habsburgo de España tuvieron, entre otras potencias, con el Imperio otomano.

\section{Pietro Velasco: un dominico renegado en la armada del Gran Turco}

Pietro Velasco vivió por lo menos cuatro vidas: la primera, su infancia y juventud, en el Reino de Nápoles; la segunda, como cautivo cristiano en poder de moros; la tercera, como renegado y marinero al servicio del Gran Turco; y la cuarta, como renegado arrepentido al servicio de la "causa cristiana", entre Toscana y España. ${ }^{12}$

Aunque no sepamos casi nada de los años juveniles de este renegado, es importante señalar que aparte de cristiano bautizado - condición necesa-

10 LEWIS, Bernard (1990), Race and Slavery in the Middle East: an Historical Enquiry, Oxford University Press, Nueva York, p. 6; y GORDON, Murray (1987), L'Esclavage dans le monde arabe: Vlle-XXe siècle, Robert Laffont, París, p. 39.

11 Evidentemente, para cada historia de integración y deslumbrante éxito, existen miles de historias más discretas, trayectorias vitales destinadas a dejar huellas menos profundas en los archivos del espionaje europeo. No lo estamos olvidando, pero asumir la perspectiva de la Monarquía Hispana significa, en este caso, dirigir nuestra atención a aquellos musulmanes nuevos que han alcanzado el éxito y el poder necesarios para que Madrid llegue a considerarles como interlocutores ventajosos.

12 La presente reconstrucción biográfica recoge fuentes documentales procedentes de distintos archivos, siendo los más destacados el Archivo General de Simancas (sección Estado-Génova) y el Archivio Segreto Vaticano (Fondo Borghese). 
ria para que un renegado sea tal, especialmente desde el punto de vista inquisitorial - antes de apostatar Velasco es también un hombre de religión a todos los efectos, tonsurado en Nápoles por la Orden de Santo Domingo. En el umbral del siglo XVI, las conversiones de hombres religiosos a la fe islámica son una cuestión tan frecuente como polémica, como bien evidencia el caso Pedro Pacheco, hijo del virrey siciliano Villena y contemporáneo de Velasco, que en 1609 reniega junto con su secretario causando un escándalo que hasta llama la atención de Paolo Sarpi. ${ }^{13}$ Efectivamente, las apostasías protagonizadas por miembros del clero tienen implicaciones muy problemáticas desde varios puntos de vista. Para limitar el escándalo causado por estas particulares abjuraciones, se opta frecuentemente para un trato especialmente misericordioso - siendo en este sentido muy paradigmático el caso del joven Pacheco - y se facilita enormemente la rehabilitación de los clérigos perdidos, que a menudo hasta recuperan el hábito religioso tras abjurar del error cometido en tierra de moros. Como veremos, este será también el caso de Pietro Velasco, amparado en su retractación por un patrón tan potente como interesado en agilizar su reinserción social en tierras cristianas.

La segunda vida de Velasco empieza alrededor de 1583, cuando este se ve, en circunstancias poco claras, "cautivado de moros» y llevado forzosamente a Turquía. ${ }^{14}$ Ahí, probablemente tras un tiempo a la espera de un rescate que no termina de llegar, Velasco se convierte a la religión islámica y empieza a dedicarse al oficio de marinero y corsario.

$\mathrm{Si}$ bien nuestras fuentes son poco elocuentes con respecto a los arranques de la carrera pirática de Velasco, lo que sí nos transmiten con gran claridad es que la inserción de este cautivo en el mundo turco-berberisco es muy exitosa, tanto a nivel personal y social (en Turquía Velasco se casa y tiene hijos), ${ }^{15}$ como a nivel económico y profesional.

13 En una carta para Francesco Priuli, embajador veneciano en Praga, Sarpi se declara muy sorprendido porque los beneficios eclesiásticos que corresponden al joven Pacheco no se revocan tras su apostasía sino que se transfieren sencillamente a su padre (carta fechada 6 de octubre de 1609). MONTICONE, Alberto (1961), Paolo Sarpi, Lettere ai gallicani. Edizione critica, saggio introduttivo e note a cura di Boris Ulianich, Franz Steiner Verlag, Wiesbaden, p. 116; PRETO, Paolo (2010), I servizi segreti di Venezia: spionaggio e controspionaggio ai tempi della Serenissima, II Saggiatore, Milán, p. 348; y PRETO, Paolo (2013), Venezia e i Turchi, Viella, Roma, pp. 197-198.

14 Juan Vivas comenta en 1608 que han pasado 25 años desde el momento de su apresamiento («fue cautivo de moros habrá 25 años»). Archivo General de Simancas (en adelante AGS), Estado, L. 1932, ff. 512r-512v. 
Y efectivamente, en Levante Velasco no se limita a la navegación corsaria de nivel "medio-bajo", finalizada a la exclusiva acumulación de riquezas, sino que asciende a las altas esferas de la flota otomana. Durante «14 años continuos» navega al lado del "general Cigala», ${ }^{16}$ un renegado de origen italiano que en 1591 accede al cargo de Kapudan Pasha y da pie a una serie de campañas militares muy agresivas contra Persia y contra los Habsburgo de Austria, así como a numerosas expediciones de ataque a las costas del Mezzogiorno de Italia.

Aunque se encuentre fuera de los límites del Mar Adriático, Cigala lleva su flota a Calabria en más de una ocasión. En septiembre de 1594 sus hombres saquean e incendian algunas zonas de Reggio Calabria. ${ }^{17}$ Asimismo, es en aguas calabresas cuando en otoño de 1598 Scipione Cigala se reúne con su madre Lucrezia, 37 años después de su cautiverio. Para acudir al encuentro, que se realiza bajo la supervisión del virrey de Sicilia, el Kapudan Pasha zarpa desde Estambul con más de cincuenta naves y con esta pequeña armada consigue cruzar el estrecho de Messina sin encontrar grandes resistencias. Sin embargo, la opinión pública teme otro ataque a Calabria y sigue con gran preocupación a los movimientos de Cigala. ${ }^{18}$

Es importante destacar que el gran valor añadido que renegados como Pietro Velasco pueden ofrecer a los corsarios turco-berberiscos, por encima de todo talento personal, es precisamente su conocimiento del territorio enemigo. No es infrecuente que los cristianos de Alá pongan sus competencias lingüísticas y geográficas a disposición del Turco para facilitar las incursiones encaminadas a dañar las costas de Italia, Francia y España. Es una operación que tiene sus riesgos - no faltan los apóstatas que aprovechan de la cercanía con sus tierras natales para desertar y fugarse - pero los beneficios son considerables y el juego se mantiene en pie a pesar de todo.

15 «Habiendo renegado se casó y tuvo hijos en la parte de Levante» (Estado, L. 1932, ff. $512 \mathrm{r}-512 \mathrm{v})$. La creación de vínculos familiares es un índice de integración social muy importante, es por este motivo que las fuentes del Santo Oficio siempre destacan mucho las trayectorias matrimoniales y parentales de los cristianos de Alá. GONZÁLEZ-RAYMOND, La Croix et le Croissant, pp. 168-174.

16 AGS, Estado, L. 1932, ff. 512r-512v, 28 agosto 1608.

17 Sobre este episodio: TÜRKÇELIK, Evrim (2012), Cigalazade Yusuf Sinan Pasha y el Mediterráneo entre 1591-1606. Tesis Doctoral, Universidad Autónoma de Madrid, Madrid, pp. 197. 203.

18 Sobre la reunión entre Scipione Cigala y su madre: TÜRKÇELIK, Cigalazade Yusuf Sinan Pasha p. 309; y SOLA, Emilio (2007), La Conjura de Campanela, Turpin, Madrid, p. 68. Para una bibliografía completa de los historiadores sicilianos y napolitanos que nararron el evento en sus crónicas. Veáse también la tesis de Evrim Türkçeli. 
Aunque no tengamos pruebas concretas de ello, no es absurdo imaginar que la opinión de Pietro Velasco contribuya a configurar las mencionadas expediciones. Velasco, que ha sido cautivado en edad suficientemente madura como para haber sido ya tonsurado, no puede haber olvidado su tierra natal. Tanto la armada turca como los servicios de inteligencia de la Monarquía hispánica, aunque, como veremos, de formas opuestas, se aprovecharán de la procedencia de Velasco en beneficio propio.

\section{HUYendo de TuRquía}

Pese al éxito y a la fortuna acumulada en Turquía, a principios del siglo XVII Velasco decide abandonar la flota otomana y entrar al servicio de Florencia. Mientras todas las fuentes consultadas -tanto las hispanas como las italianas- silencian las circunstancias de su conversión al islam, es gracias a las segundas -concretamente a la correspondencia que el nuncio Antonio Grimani intercambió con Paulo V- que sabemos algo más sobre las circunstancias de su regreso a tierras cristianas y las de su reconciliación con la Iglesia. ${ }^{19}$

Por lo que respecta a la llegada de Velasco a Italia, esta es el resultado de una fuga muy aventurera. Adaptándose totalmente a la retórica del arrepentimiento religioso -absolutamente omnipresente en las fuentes hispanas del siglo XVI-XVII que hablan del reclutamiento de renegados por parte de la Monarquía Hispánica- ${ }^{20}$ en 1608 el embajador Juan Vivas describe la deserción de Velasco como el fruto de una iluminación espiritual - el segundo de Cigalazade deja Estambul «tocado por Nuestro Señor en el corazón», sin más.

Afortunadamente, resulta más completa la descripción que nos proporciona en 1606 la correspondencia de Antonio Grimani, nuncio apostólico en la Florencia de Fernando de Médicis. Si bien también apoyándose en la retórica del arrepentimiento religioso, Grimani nos devuelve el apasionante relato de una fuga empezada en el puerto de Quíos, fastidiada por tormentas, dificultada por la persecución de una escuadra de cincuenta jenízaros, ${ }^{21}$ y finalizada en Ancona, por supuesto gracias al amparo divino (ofrecido en este caso por la Virgen de Loreto). ${ }^{22}$

19 No olvidemos que, en los territorios sujetos a la jurisdicción del Santo Oficio (tanto español, como romano), la reconciliación por vía inquisitorial es teóricamente obligatoria para los cristianos de Alá.

20 Este es uno de los temas centrales de mi investigación doctoral. Profundizo más en OLDRATI, Valentina (2018), "Renegades and the Habsburg secret services in the aftermath of Lepanto: Haci Murad and the Algerian threat as a case study", Journal of Iberian and Latin American Studies, $n^{\circ} 24, \mathrm{pp} .7-26$. 
Dejando a un lado la cuestión de los remordimientos, insondable como todo tema espiritual, lo que sí podemos decir con certeza es que Velasco se va de Estambul dejando atrás todos sus bienes, hecho que cobraría bastante más sentido en el marco de una fuga repentina con trasfondos políticos, como por ejemplo el declive político y militar que sufre su patrón Cigalazade durante la última etapa de su vida.

Efectivamente, y como bien ha sido explicado por Evrim Türkçelik, en la segunda mitad de la década de 1590 la carrera del Cigala entra en una etapa crítica a raíz de la muerte del sultán Murad III (1595) y de la consiguiente reconfiguración de los equilibrios internos de la Corte otomana. ${ }^{23}$ La situación es fluctuante, como demuestra el entusiasmo con que se recibe la victoria obtenida por Cigalazade contra el archiduque Maximiliano en Hungría (1596), ${ }^{24}$ pero el clima general es bastante tenso. Para consolidar su poder, el nuevo Gran Visir y la madre del nuevo Sultán Mehmed III hacen lo posible para desacreditar a Cigalazade, y lo hacen insistiendo especialmente en sus orígenes cristianos y en sus sospechosas amistades y relaciones con cristianos (in primis con su hermano Carlo) y con cristianos renegados. ${ }^{25}$

No es absurdo imaginar que este afán recriminatorio tenga un impacto negativo en la vida y en la carrera de hombres como Pietro Velasco. A

21 Lo cual probaría la importancia alcanzada por Velasco dentro de la armada turca. Sin embargo, podría tratarse de una exageración.

22 Archivio Segreto Vaticano (en adelante ASV), Fondo Borghese, S. II, 303, ff. 71 r-71 v.

23 TÜRKÇELIK, Cigalazade Yusuf Sinan Pasha, pp. 205-256; y TÜRKÇELIK (2013), “El renegado "El Cigala' y la política mediterránea", Desperta Ferro. Historia Moderna, n 6, pp. 40-43.

24 El Sultán premia a Cigalazade con el prestigioso título de Gran Vizir. Cigalazade mantiene el título durante unos meses, pero la rebelión de las tropas kurdas y las conspiraciones de palacio llevan al sultán a revocar su nombramiento. Al cabo de poco tiempo, Cigala será nuevamente nombrado Gran Vizir y también General de la mar. Cfr.: BUONFIGLIO COSTANZO, Giuseppe (1604), Prima [e secundi] parte dell'Historia siciliana, Bonifacio Ciera, Venecia, p. 678; y MONTUORO, Domenico (2009), "I Cigala, una famiglia feudale tra Genova, Sicilia, Turchia e Calabria", Mediterranea: Richerche Storiche, n 6, p. 289.

25 Dice la leyenda que Lucrezia, madre de Scipione e hija del bey de Castelnuovo, se había casado con el padre de Cigalazade tras haber sido apresada con ocasión de la toma del puerto montenegrino en 1538. Los orígenes mixtos de Scipione, pese a ser un poco turbios para los estándares españoles de limpieza de sangre, nunca representan un problema en las fuentes cristianas. Sin embargo, el hecho de que la madre del Cigalazade haya abandonado el islam en favor del cristianismo es bastante mal visto en Turquía, y los detractores de Cicala no pierden ocasión para preguntarse públicamente si la conversión de Lucrezia no pueda exponer el almirante al peligro de convertirse a su vez a la religión cristiana. Cfr.: OCAKAÇAN, Levent Kaya (2015), "Cigalazade Yusuf Sinan Pasha (C. 1545-1606)", Mediterranea: Richerche Storiche, Palermo, $n^{\circ} 12$, pp. 325-340; y MAS, Albert (1967), Les Turcs dans la littérature espagnole du Siècle d'Or: recherches sur l'évolution d'un thème littéraire, Centre de Recherches Hispaniques, París, Vol. 2, pp. 272-273. 
veces por conveniencia y a veces huyendo de un peligro inminente, no falta quien tome el camino de la defección y busque nuevos patrones a los cuales ofrecer sus competencias y servicios.

\section{Al servicio del Gran Duque}

Muy interesante es también la cuestión de la reconciliación de Pietro Velasco, un trámite de naturaleza política que suscita cierto debate entre el Papado, Fernando I, y el tribunal inquisitorial de la capital medicea.

Aunque en la Florencia de comienzos del siglo XVII exista un tribunal perfectamente capacitado para reconciliar a todos los cristianos de Alá, ${ }^{26} \mathrm{Ve}$ lasco consigue canjear sus cargos inquisitoriales por un simple peregrinaje penitencial a Roma (1606). Gracias a las presiones del gran duque, transmitidas a Roma con la mediación del nuncio Grimani, el pontífice hace expedir un salvoconducto con validez de seis meses, en virtud del cual Velasco alcanza la capital, hace penitencia y es readmitido en la Orden dominica, todo ello sin pasar por los inconvenientes normalmente causados por un proceso inquisitorial:

Questo fu frate di San Domenico et per rimorso di coscienza è voluto ritornare alla vera fede, è pronto di venir a Roma ad humiliarsi et a ricevere ogni penitenza et a ripigliarsi l'abito della sua Religione et perciò ha procurato un salvocondotto della Sacra Congregazione di venire a Roma dentro a sei mesi del quale io ho veduto copia. Hora mentre si aspettava questo salvocondotto il Padre Inquisitore di Firenze è stato a Pisa, et avendo fatto personalmente istanza di avere costui nelle mani, Sua Altezza gli ha detto che lo terrà in Castello fin a nuovo ordine da Roma, et che gli pareva strano che contro chi spontaneamente veniva ad umiliarsi [si procedesse] con questo rigore, et che questo era un levare l'animo agli altri rinnegati che avessero voglia di ritornare nel gremio della Chiesa, et dopo questa istanza dell'inquisitore venne il salvocondotto. Mi ha perció Sua Altezza ostinatamente pregato che supplichi Sua Santità che si contenti che questo uomo faccia qui l'abiurazione in mano dell'Inquisizione e di Firenze et di Pisa, con tutto quel di più che sarà necessario per tale effetto, et che farà ogni penitenza et ripiglierà l'abito, et questo

26 Sobre el tribunal de Florencia: PROSPERI, Adriano (2011), "Firenze", en PROSPERI, Adriano, LAVENIA, Vincenzo y TEDESCHI, John (coords.). Dizionario storico dell'Inquisizione, Edizioni Normale di Pisa, Pisa, Vol. 2, pp. 605-607. 
desidera Sua Altezza per due rispetti, l'uno per non spaventare gli altri rinnegati di ritornare alla fede, l'altro perché ha tanto bisogno del consiglio di costui per servizio dell'impresa contro Turchi, che concorrono ante il servizio della Cristianità. ${ }^{27}$

Es interesante notar que, para deslegitimar las peticiones del inquisidor de Florencia -cargo que entre 1606 y 1615 fue ocupado por el franciscano Cornelio Priatoni da Monza- Grimani atribuye a Fernando I un discurso sobre la importancia de la templanza inquisitorial ante el fenómeno de los cristianos de Alá que a principio del siglo XVII está ya muy extendido en todos los territorios que están sujetos a la jurisdicción del Santo Oficio, ambos romano y español. Si el objetivo de la lglesia es favorecer el regreso a tierras cristianas del mayor número de renegados posible -argumenta Grimani a través del gran duque- causar inútiles inconvenientes a un penitente espontáneo y tan bien dispuesto como Velasco no puede ser sino una decisión corta de miras y contraproducente. ${ }^{28}$

Como también subraya Francesco Vitali, estudioso de la Nunciatura Apostólica en la Florencia de 1587-1609, Velasco es el hombre adecuado para convertir en realidad el sueño de una expedición naval antiotomana bajo la égida de las galeras de Toscana, lo cual explica la gran obstinación mostrada por su reclutador frente a todo inconveniente práctico o burocrático que ponga en riesgo su rápida reinserción en la sociedad italiana. ${ }^{29}$

Fernando I mira hacia Oriente para fortalecer la posición de Toscana y de la Orden de Santo Stefano dentro del escenario político italiano, ${ }^{30}$ y Chipre es el objetivo militar perfecto para ello. Caída en manos otomanas en 1571, Chipre se encuentra efectivamente en una situación estratégica inmejorable tanto de cara a una eventual recuperación de los Santos Luga-

\section{ASV, Fondo Borghese, S. II, 303, ff. 71 r-71v.}

28 Sobre el tema de la spontanea comparitio, cfr.: BRAMBILLA, Elena (2006), La giustizia intollerante. Inquisizioni e tribunali confessionali in Europa (secoli IV-XVIII), Carocci, Roma, pp. 6871; y BRAMBILLA, Elena (2000), Alle origini del sant'Uffizio. Penitenza, confessione e giustizia spirituale dal Medioevo al XVI secolo, II Mulino, Bolonia, pp. 381-402.

29 Velasco aparece en el párrafo que Vitali dedica a la nunciatura de Antonio Grimani en Florencia (1605-1616), aunque sin que el autor haga referencia al sucesivo pasaje de este capitán al servicio de Felipe III. Sin embargo, las detalladas referencias de archivo ofrecidas por Vitali nos han permitido acudir a las fuentes vaticanas y encontrar informaciones de gran interés sobre la reconciliación de reclutas renegadas de alto rango en el contexto de las relaciones entre Roma y la Toscana de los Médicis. Cfr.: VITALI, Francesco (2017), I nunzi pontifici nella Firenze di Ferdinando I (1587-1609), Nuova Cultura, Roma, pp. 156-170.

30 Incluso después del fracaso chipriota, Toscana no dejará de mirar hacia Levante. En septiembre de 1607 los caballeros de Santo Stefano, encabezados por Silvio Piccolomini, conquistarán Bona (actual Annaba, en el noreste de Argelia). En 1613 Fernando I acogerá en Florencia 
res, como para llevar la guerra al interior de Turquía. ${ }^{31}$ Al mismo tiempo, el mito del martirio de Marcantonio Bragadino, ${ }^{32}$ desollado vivo en plaza de Famagusta, y los constantes llamamientos que la población cristiana de Chipre hace a Toscana, España y a los duques de Saboya confieren a la Reconquista de la isla un altísimo valor simbólico. ${ }^{33}$

No es difícil entender porque el gran duque, que ha recibido «infiniti servizi de un Capitano Pietro Velasco calabrese nell'imprese delle sue galere» y que espera recibir muchos otros "ne i disigni che ha contro il Turco», ${ }^{34} \mathrm{sim}$ plemente no vea con buen ojo que algo frene la salida de Velasco a la mar. En cualquier caso, el fuerte interés que Fernando I tiene en la rápida e indolora rehabilitación de Velasco no debería engañarnos. Las relaciones entre los dos son borrascosas y desde el primer momento, tanto en lo personal como en ámbito militar.

Las primeras fricciones surgen incluso antes de que Velasco viaje a Roma, cuando el gran duque descubre, por una carta del cardenal Arrigoni, ${ }^{35}$ que tras llegar a Italia el capitán Velasco se ha casado secretamente con una mujer griega, hecho incompatible con el estatuto sacerdotal que el

al desertor Fakhreddin, príncipe de la provincia libanesa de Sidon. Tras pasar un tiempo en, Fakhreddin moverá su residencia a Messina (1615) y luego a Nápoles (1616), donde residirá hasta volver a Líbano en 1618. Incluso tras regresar a su tierra natal, Fakhreddin no dejará de vender informaciones a sus contactos italianos e hispanos. El propio emir ha escrito una memoria de su viaje a Italia, que ha sido traducido al italiano en 1936: CARALI, P. Paolo (1936), "Soggiorno di Fakhr ad-dīn al-Ma'nī in Toscana, Sicilia e Napoli e la sua visita a Malta (1613-1618)", Annali del Istituto Superiore Orientale di Napoli, n 4.

31 Sobre Chipre a comienzos del siglo XVII y la expedición toscana de 1607: HILL, George (1952), A History of Cyprus: The Ottoman Province, the British Colony, 1571-1948 (Vol. 4), Cambridge University Press, Cambridge; FLORISTÁN, José M. (1988), Fuentes para la política oriental de los Austrias. La documentación griega del Archivo de Simancas (1571-1621), Universidad de León, León, pp. 693-737; FLORISTÁN, José M. (2016), "Relación de la empresa de Famagusta (1607)", Archivo de la Frontera, <http://www.archivodelafrontera.com/ wp-content/uploads/2016/09/1607-EMPRESA-DE-FAMAGUSTA-JM-Florist\%C3\%A ln.pdf>; HADIIANASTASIS, Marios (2014), "Corsair tactics and lofty ideals: the 1607 Tuscan raid on Cyprus", en WALSH, Michael (ed.), Historic Famagusta: A Millennium in Words and Images, Cambridge Scholars Publishing, Cambridge, pp. 22-36; y GEMIGNANI, Marco (2003), "The navies of the Medici: The florentine navy and the navy of the sacred Military order of saint stephen, 1547-1648", en HATTENDORF, John y UNGER, Richard (eds.), War at Sea in the Middle Ages and the Renaissance, Boydell press, Woodbridge, pp. 169-185.

32 Rector de la fortaleza en el día de su conquista otomana. Muere en fecha 17 de agosto de 1571.

33 HADJIANASTASIS, "Corsair tactics and lofty ideals", p. 24.

34 ASV, Fondo Borghese, S. II, 303, ff. $71 \mathrm{r}-71 \mathrm{v}$.

35 A partir de 1605 Pompeo Arrigoni fue el secretario de la Sagrada Congregación de la Romana y Universal Inquisición. 
ex-renegado pretende recuperar a través del perdón papal. Irritado por los subterfugios de su criado, Fernando I le encarcela.

Grimani, como siempre atento observador de la Corte florentina y de los humores de su carismático líder, se sorprende por esta "repentina mutazione» hasta el punto de declararse «il più confuso vomo del mondo», y comunica prontamente con Roma sobre el encarcelamiento de Velasco. Disgustado, pero consciente de las cualidades militares de su recluta, el gran duque termina liberando a Velasco, que se dirige hacia los estados pontificios tal y como pactado ("Sua Altezza era tanto sdegnata contro costui per avere ... inteso che aveva sposato una greca ch'essendogli fatta istanza che lo mandasse a Roma, aveva volute anteporre la soddisfazione di Sua Santità ai propri interessi»). ${ }^{36}$ De la mujer griega con la cual Velasco se ha casado en Florencia no tenemos más trazas. ${ }^{37}$

Y los inconvenientes generados por Velasco no se limitan a su irregular conducta privada: su indocilidad llega a causar problemas también de orden público, como nos muestra la noticia de una pelea ocurrida entre Velasco y Francesco Mancini da Montepulciano, caballero de Malta, con ocasión de la cual nuestro ex-renegado resulta herido por su adversario (1607). ${ }^{38}$

Superadas las dificultades en el plano personal, empiezan las dificultades de tipo militar. Según leemos en la «Relación de lo que an hecho en Levante las galeras del gran duque y el sucesso de Famagusta», editada y publicada por José Manuel Floristán Imizcoz, Fernando I deja de lado todo desacuerdo y resuelve poner a Pietro Velasco a la cabeza de uno de los navíos toscanos que intentan tomar Famagusta en mayo de 1607 («marciava di vanguardia il capitano Pietro Velasco con un petardo in compagnia di 25 huomini»). ${ }^{39}$

36 ASV, Fondo Borghese, S. II, 303, ff. 76r-76v; ASV, Fondo Borghese, S. II, 303, f. 77r.

37 En su informe para Felipe III, Juan Vivas comenta que Fernando manda que la mujer de Velasco sea bautizada y metida a monja. Sin embargo, se refiere a la mujer - turca de nacimiento con la cual Velasco se ha casado en Turquía y con la cual ha huido de Quíos. La boda que provoca la indignación de Fernando es posterior, tiene lugar en Toscana e involucra con toda probabilidad a una mujer cristiana. AGS, Estado, L. 1932, ff. 512r-512v.

38 CAPPONI, Gino (1845), Catalogo dei manoscritti posseduti dal Marchese Gino Capponi, Coi Tipi della Galileiana, Florencia, pp. 13-14. Estos son los títulos completos de los dos manuscritos del Fondo Capponi que refieren a Pietro Velasco, y que esperamos localizar y consultar en una futura estancia de investigación: "88. Deliberazione fatta il primo di luglio (1607), sopra il capo della Grecia, del signor Ammiraglio, Capitan Pietro Velasco et altri intendenti, sopra quello che si deve far delle galere"; "n. 89. Vari costituti nel processo formato per la rissa accaduta tra il Capitano Pietro Velasco di Calabria, ferito dal cavalier Francesco Mancini da Montepulciano (1607)". Gino Capponi fue un político e historiador florentino (1792-1876). Su biblioteca personal fue donada a la Biblioteca Nazionale di Firenze en 1879 y contiene numerosos manuscritos de gran interés histórico. 
Se trata de una posición de gran relieve si consideramos que Velasco comparte el mando con personajes del calibre de Francesco del Monte -estimado general y autor de una interesante crónica de la jornada titulada "Impresa di Famagosta" - ${ }^{40}$ y Antonio de Médicis -sobrino del propio gran duque, caballero de la Orden de Malta, y diplomático de perfil internacional. ${ }^{41} Y$ es tal vez por este motivo que el otomanista Marios Hadjianastasis confunde nuestro Pietro Velasco, ex-renegado de origen calabrés, con Pedro Téllez-Girón y Velasco, gran duque de Osuna y futuro virrey de Sicilia (1610-1616).42 Sin embargo, cabe señalar que su reconstrucción de la ofensiva medicea realizada a través de la crónica redactada por Del Monte y de fuentes procedentes del archivo de la familia Piccolomini de Siena- sigue siendo muy detallada, y permite seguir prácticamente todos los movimientos efectuado por Velasco en Famagusta. ${ }^{43}$

Pese a las grandes expectativas -Velasco había prometido entregar Chipre en virtud de su excelente conocimiento del Mediterráneo oriental, por lo menos según las declaraciones de Juan Vivas («[dijo] que le diera a Cipro si hizieran lo que dixo»|-44 la misión chipriota falla míseramente. Para empeorar las cosas, una de las causas del fracaso florentino -al margen de la gran capacidad de reacción mostrada por el ejército turco- ${ }^{45}$ es precisa-

39 AGS, Estado, L. 1162, ff. 200-201 (la relación se remite a Madrid desde Mesina, junto con una carta del virrey de Sicilia Juan Fernández Pacheco, duque de Escalona). Publicado por FLORISTÁN, Fuentes para la política oriental de los Austrias, pp. 716-721.

40 Fuente citada por: HADJIANASTASIS, "Corsair tactics and lofty ideals", p. 24. Referencia de archivo: Biblioteca Comunale degli Intronati de Siena, K. II.16, ff. 132-176 ("Impresa di Famagosta: Giustificatione dell'lllustrissimo Signore Francesco del Monte").

41 Sobre la misión: ASV, Fondo Borghese, S. II, 302, f. 175r. Nótese que este mismo volumen contiene varias noticias sobre la preparación y el fracaso de la misión.

42 Así escribe Hadjianastasis en el artículo arriba citado: "There were also other noble participants: Don Antonio de' Medici, the late Granduke Francesco I's son with his ten mistress (and future wife) Bianca Cappello, was part of the expedition; so was Pietro Velasco, the future Duke of Osuna and Viceroy of Sicily». HADIIANASTASIS, "Corsair tactics and lofty ideals", p. 26.

43 Por ejemplo, gracias a la reconstrucción de Hadjianastasis sabemos que Velasco llega a Chipre al mando de las petardas (p. 27). Más tarde, cuando se decide atacar a la fortaleza escalando su muralla, la compañía de Pietro Velasco se moviliza en apoyo a la de Pietro Viscardo, encargado de penetrar por el lado sur del castillo (p. 29). Pasando ya a la etapa final del asalto, Hadjianastasis ubica a Velasco entre los hombres que atacan la fachada noreste del castillo, donde la contemporánea reagrupación de cuatro compañías genera una parálisis que termina colocando a las tropas toscanas totalmente a la merced del fuego enemigo, (p. 31). Finalmente, tras retirarse la armada en Pannaia (una bahía ubicada a 10 millas de Famagusta), Velasco muestra ser ferozmente partidario de mover otro ataque a la fortaleza. Para ello promete reunir a un ejército de griegos, pero la misión fracasa (pp. 33-34).

44 AGS, Estado, L. 1932, ff. 512r-512v. 
mente la mala planificación del ataque, debida a un total desconocimiento de las murallas de Famagusta, ${ }^{46}$ y a la tardía llegada de las galeras de Montecuccoli. ${ }^{47}$

Consideradas las promesas hechas por Velasco, no es improbable que sea el fracaso de la misión la razón de la ruptura entre nuestro capitán y Florencia. Una carta que Grimani envía a Paulo V el 4 de agosto de 1607, y en la cual se habla de una posible fuga de Velasco a Calabria, parece confirmar nuestras sospechas: el fracaso chipriota ha quitado credibilidad a Velasco, y su largo historial de cambios de bando no hace sino empeorar la situación. ${ }^{48}$

\section{De Toscana a la Monarquía hispánica}

Legítimos $O$ no, el autoritarismo y la constante vigilancia ejercitados por Fernando tras Chipre ponen duramente a prueba a Velasco, que en 1608 decide abandonar Florencia en favor España. La notoria receptividad mostrada por la Monarquía hispánica hacia el reclutamiento de renegados y ex-renegados, siempre y cuando fuesen colaborativos y "de buen rango", convierten a Felipe III en un patrón ideal para los objetivos de nuestro capitán, cuyas propiedades e hijas han sido secuestradas de forma preventiva por el tercer gran duque. ${ }^{49}$

Las tentativas autonomistas introducidas por el "francesista" Fernando I y las consecuentes tensiones en que se traducen en las relaciones Habsburgo-Mediceas, ${ }^{50}$ deben facilitar aún más el proceso decisorio del ex-renegado, seguro de encontrar en Madrid unos patrones a la altura de su potencial.

45 Aunque se realice de madrugada, la llegada de las galeras de Florencia no pasa desapercibida, con lo cual el desembarco no puede contar con el factor sorpresa.

46 Se subestima la altura de la muralla exterior de Famagusta (de hecho, las escaleras empleadas para subirla resultan ser demasiado cortas) y se desatiende la existencia de una segunda muralla interior.

47 Alessandro Montecuccoli había sido lugarteniente de los soldados de Siena bajo Alessandro I de Médicis. Al asalto de Famagusta participó con cinco barcos. Véase FLORISTÁN, "Relación de la empresa de Famagusta (1607)".

48 ASV, Fondo Borghese, S. II, 306, f. 40v.

49 Así se describe la situación de Velasco a Felipe III: «le hará mucho servir a Vuestra Magestad y salir del Duque porque le ha tomado la hacienda y las hijas de temor que no se le vaya». Ibidem.

50 Fernando I es mucho más audaz que su padre Cosimo, por lo menos en su relación con Espa- 
Para este efecto, y estando en Génova para comprar un barco, Pietro decide entonces ponerse en contacto con el embajador de España, Juan Vivas de Cañamás. La proximidad geográfica con Toscana y la vinculación política con Madrid convierten la Repubblica en un sitio ideal para un potencial desertor como Velasco. ${ }^{51}$ Por añadidura, Vivas es un atento observador del escenario chipriota, ${ }^{52}$ y ha consagrado ya notables esfuerzos a neutralizar la amenaza turco-berberisca en el Mediterráneo occidental, vigilando los movimientos de los moriscos de Valencia y Cataluña e informando constantemente a Madrid al respecto. ${ }^{53}$

$Y$ efectivamente Vivas cumple con las expectativas: entusiasmado, escribe enseguida a Felipe III con el objetivo de recomendar el reclutamiento de Velasco, que mientras tanto sigue oficialmente a sueldo toscano. ${ }^{54}$

Aparte de describir las cualidades del capitán y de omitir casi toda referencia a su historial de traiciones político-religiosas, ${ }^{55}$ nos parece interesante que el mensaje de Vivas haga referencia a la procedencia del ex-apóstata subrayando, de forma sutil pero no casual, su condición de súbdito de la

ña. No solamente interviene en las guerras de religión francesas al lado del protestante Enrique IV, sino que también consigue que este se case con su sobrina Maria de Médicis en 1599. Sobre Fernando I de Médicis y el giro al "francesismo" de la política toscana, cfr.: GONZÁLEZ TALAVERA, Blanca (2011), Presencia y mecenazgo español en la Florencia Medicea: de Cosme I a Fernando I. Tesis Doctoral, Universidad de Granada, Granada, pp. 74-78; HANLON, Gregory (1998), The Twilight of a Military Tradition: Italian Aristocrats And European Conflicts, 1560-1800, Routledge, Londres, pp. 39-40.

51 Manuel Herrero Sánchez se ha servido de la eficaz fórmula "agregado hispanogenovés" para describir las relaciones entre la República de Génova y la Monarquía Hispana durante los siglos XVI y XVII: HERRERO SÁNCHEZ, Manuel (2001), "Una república mercantil en la órbita de la Monarquía Católica (1528-1684)", en ANATRA, Bruno y MANCONI, Francesco (eds.), Sardegna, Spagna e Stati Italiani nell'Età di Carlo V, Carocci, Roma, pp. 183-200.

52 Vivas escribe varias cartas a Felipe III sobre la fallida insurrección chipriota de 1606. En ellas afirma que la población local podría fácilmente aliarse con España con tal de emanciparse del Turco. Cfr.: LUBIN, Matthew (2011), Aftermath of War: Cypriot Christians and Mediterranean Geopolitics, 1571-1625. Tesis Doctoral, The University of North Carolina at Chapel Hill, Chapel Hill, p. 134. Fuentes citadas: AGS, Estado, L. 1434, docs. 47, 102, 117.

53 Vivas escribió varias cartas a Felipe III para informarle sobre los moriscos que hacían escala en Francia antes de zarpar hacia Argel. Cfr.: GIL HERRERA, Jorge y BERNABÉ PONS, Luís F (2014), "The Moriscos Outside Spain. Routes and Financing", en GARCÍA-ARENAL, Mercedes y WIEGERS, Gerard (eds.), The Expulsion of the Moriscos from Spain: a Mediterranean diaspora, Brill, Leiden, pp. 222-223.

54 Con fecha 6 de julio de 1608 Juan Vivas había remitido a Felipe III las siguientes informaciones: «El gran duque también sale con su armadilla y ha enviado delante cuatro galeras con fray Pedro Velasco, llevarán en todo diez galeras y cosa de quince bajeles redondos sin otros pequeños». Se deduce que Velasco era un personaje conocido dentro del panorama militar mediterráneo. AGS, Estado, L. 1434, doc. 121. 
Corona, nacido en Calabria, y además «hijo de español natural de esta villa de Madrid».56

Como súbdito de Felipe III, Velasco tiene una obligación moral no solamente hacia su rey, sino también hacia la causa de la verdadera fe: dos obligaciones intrínsecamente ligadas entre si, hecho que los servicios de inteligencia de la Monarquía hispánica difícilmente se olvidan de destacar cuando intentan el reclutamiento de un renegado entre los siglos XVI y XVII. Es así que el reclutamiento de Velasco, elevado a justo retorno de una oveja descarriada, sirve a España para fastidiar no solamente al Turco, sino también a los Médicis e, indirectamente, a un pez incluso más gordo: la Francia de Enrique IV.

\section{FORMAS DE PATRONAZGO... Y DE MUTUO ESTORBO}

En el Mediterráneo del siglo XVII la promoción del talento, se ha visto claramente, es una práctica "trans-imperial". Lo es en cuanto común a todas las formaciones políticas dotadas de medios económicos adecuados, y lo es en cuanto subproducto de unas rivalidades que ven en la sustracción de recursos humanos una estrategia bélica doblemente triunfadora, por su capacidad de usar la fuerza del enemigo -en este caso en forma de capital humano- en su contra.

La armada otomana forma y da responsabilidades militares al joven Velasco, nacido cristiano y fuera del imperio, Florencia lo recluta cuando ya es un marinero experto y deseoso de cambiar de aires, y lo mismo se proponen hacer los Habsburgo de España cuando la historia se repite. En cada una de estas coyunturas, el reclutamiento de Pietro Velasco corresponde a una voluntad de "promoción del talento", pero también puede verse como un efecto secundario de rivalidades políticas y militares: se trata, para emplear una fórmula que sintetice nuestra interpretación, de estrategias de "asimilación antagonista", encaminadas a ofrecer un beneficio interno con el complemento de un estorbo externo.

Especialmente interesante es, en este sentido, la cuestión del "ascensor social otomano". El historiador húngaro Gabor Ágoston habla del uso de expertos extranjeros por parte del Imperio Otomano a través de la fórmula

55 Fuera de comentar que «aviendo renegado se cassó y tuvo hijos en la parte de Levante». Para lo que atañe a las cualidades de Velasco: «se tiene por cierto no aver en la christiandad hombre mas plático en la mar contra los Turcos, pues demas de saver los puertos y plazas sabe las costumbres y el modo en que se ha de navegar y se ha de llevar una armada Real».

[AGS, Estado, L. 1932, ff. 512r-512v.]

56 Ibidem. 
"the human pool of military acculturation" ("la reserva humana de aculturación militar"), ${ }^{57}$ evidenciando como ya a partir de la época de Mehmed II (1432-1481) el imperio dedicó importantes recursos a quitar a sus enemigos (o importar, según el enfoque) expertos del arte militar - capitanes y soldados, pero también autores de tratados, ingenieros, y artesanos -y como su contribución se acompaña frecuentemente a la conversión religiosa, hecho en ocasiones voluntario, pero mucho más a menudo resultado de la manumisión, a su vez medio de salida desde el cautiverio.

El camino que llevó Velasco del cautiverio a las altas esferas del ejército otomano, pasando por la apostasía, coincide efectivamente con la gran mayoría de los renegados que negociaron la venta de servicios a potencias cristianas. Los ejemplos abundan.

Uchalí, probablemente el renegado más conocido de todos los tiempos, pasa de ser cautivo del griego Khayr al-Dīn Barbarossa, a yerno y protegido del convertido calabrés Jafer Rais, su segundo amo. Gracias a una espectacular carrera militar, Uchalí termina ascendiendo a los cargos más altos de la administración otomana, entre ellos los de gobernador de Argel (1568) y de Gran Almirante de la armada otomana (1571)..$^{58}$ A partir de 1568, y durante más de veinte años, los Habsburgo de España responden a los retos planteados por Uchalí oscilando entre intricadas conspiraciones homicidas y acercamientos diplomáticos en plena regla. ${ }^{59}$

Juan de Briones, Robert Draver y Pedro/Pietro Brea - tres agentes dobles muy implicados en el famoso circulo de espías que Giovanni Margliani monta en el Estambul de Selim II - pasan de cautivos a criados de Uchalí, y como tales cooperan con los servicios de inteligencia de la Monarquía hispánica. Especialmente interesante es el caso de Brea, escribano personal de Uchalí, que filtra información sacada de la correspondencia del Kapudan Pachá a cambio dinero, títulos y rentas para sí mismo y para su familia. ${ }^{60}$

57 ÁGOSTON, Gabor (2006), Guns for the Sultan: Military Power and the Weapons Industry in the Ottoman Empire, Cambridge University Press, Cambridge, pp. 42-48.

58 Bibliografía esencial sobre este personaje: SOLA, Emilio (2011), Uchali: el calabrés tiñoso o el mito del corsario muladí en la frontera, Ediciones Bellaterra, Barcelona; SOLA, Emilio y DE LA PEÑA, José (1995), Cervantes y la Berbería: Cervantes, mundo turco-berberisco y servicios secretos en la época de Felipe II, Fondo de Cultura Económica, Madrid, pp. 72-82; y KOLOĞLU, Orhan (2007), "Renegades and the case Uluç/Kiliç Ali", en CANCILA, Rosaria (ed.), Mediterraneo in armi (secc. XV-XVIII), Quaderni di Mediterranea, Palermo, pp. 527-531.

59 GÜRKAN, Emrah Safa (2014), "Money or Your Life: The Habsburg Hunt for Uluc Ali", Studia Historica: Historia Moderna, Salamanca, n³6, pp. 121-145. 
Saliendo del ámbito estrictamente hispano, también Hasan Veneciano, nacido en Venecia con el nombre de Andreta Celeste, pasa de ser una víctima de las razzías de Dragut (1563) a ocupar los cargos beylerbey de Argel (1577-1580 y 1582-1585), de pachá de Trípoli (1587-1588), y finalmente de Kapudan Pasha (1588-1591). Una vez más, es el amparo de su apresador - y de Uchalí, a partir de 1565 - lo que le permite aprender el oficio de marinero y ascender a los más altos cargos de la administración turca. ${ }^{61}$ En las décadas de 1570 y de 1580, tanto Venecia como España invierten grandes sumas de dinero para tratar de comprar su colaboración. ${ }^{62}$

Finalmente también Scipione Cigala, el propio patrón de Velasco, pasa del cautiverio a la escuela del Serrallo, y de ahí a la armada otomana. ${ }^{63}$ Cigala, que cae víctima de corsarios turcos con tan sólo 17 años de edad, ha tenido ya bastante experiencia marítima navegando junto con su padre, el Vizconde Cigala, y lleva esta experiencia consigo en el camino de aprendizaje que emprende dentro el Enderún (la escuela imperial). ${ }^{64}$ Como bien explica Türkçelik, el padre Scipione ha dedicado toda su vida al corso contra el Turco, y por tanto «el principal valor que atesora Cigala para los otomanos es, en gran parte, su procedencia italiana sellada en la conservación de su apellido familiar en forma otomanizada», nomenclatura que permite a Estambul valerse irónica y públicamente del hijo de un servidor de la Corona en contra de la propia Monarquía. ${ }^{65}$ Décadas más tarde (concretamente entre 1593 y 1601), España devuelve el favor a Turquía intentado reclutar al Cigala por vía de su hermano, Carlo. ${ }^{66}$

60 OLDRATI, Valentina (2015), "Pedro Brea, un genovés en Constantinopla: veinte años de espionaje y contra-espionaje entre Madrid, Nápoles, Génova e Imperio otomano (1578-1596)", en SOLA, Emilio y VARRIALE, Gennaro (coords.), Detrás de las apariencias: información y espionaje (siglos XVI-XVII), Universidad de Alcalá, Alcalá de Henares, pp. 153-173.

61 Para un cuadro biográfico introductorio, cfr.: FABRIS, Antonio (1997), "Hasan 'il veneziano' tra Algeri e Costantinopoli", Quaderni di Studi Arabi, n 15 (suppl.), pp. 51 -66; y DURSTELER, Eric (2006), Venetians in Constantinople: Nation, Identity, and Coexistence in the Early Modern Mediterranean, Johns Hopkins University Press, Baltimore, pp. 123-126.

62 GÜRKAN, Emrah Safa (2016), "His Bailo's Kapudan: Conversion, Tangled Loyalties and Hasan Veneziano between Istanbul and Venice (1588-1591)", Journal of Ottoman Studies, $n^{\circ} 48$, pp. 277-319.

63 TÜRKÇELIK, Cigalazade Yusuf Sinan Pasha; IDEM, "El renegado 'El Cigala' y la política mediterránea"; OLIVA, Gaetano (1907-1908), "Sinan Bassà (Scipione Cicala) celebre rinnegato del secolo XVI: Memorie storico-critiche", Archivio Storico Messinese, Mesina, n 8/9, pp. 266-303; y OCAKAÇAN, "Cigalazade Yusuf Sinan Pasha".

64 El vizconde es apresado junto con su hijo. Sin embargo, mientras Scipione se queda en Turquía, su padre consigue rescatarse y volver a Sicilia. 
Tanto las trayectorias turcas de hombres como Uchalí - del cautiverio al éxito profesional - como las negociaciones que estos entablan con potencias cristianas como España, Venecia, y Francia, tienen en la segunda mitad del siglo XVI su época de oro. Si el siglo XVII nos ofrece menos casos de este tipo, desde luego no es porque el Mediterráneo haya cesado de ser un mar de piratas y corsarios. ${ }^{67}$ Más bien, los otomanos dejan progresivamente de ascender a cristianos a puestos de prestigio y poder - musulmanes nuevos incluidos - debido a "teologización" del discurso político que se impone de forma creciente a partir de los reinados de Solimán el Magnífico (15201566), Selim II (1566-1574), y Murad III (1574-1595). .8 Asimismo - y especialmente para lo que atañe a los cristianos de Alá - resulta cada vez más evidente que la promoción de outsiders no es garantía de lealtad, y que los vínculos de solidaridad étnico-regional terminan afectando al Imperio mucho más de lo inicialmente esperado. ${ }^{69}$

Sin embargo, en los albores del siglo XVII la promoción del talento sigue teniendo importancia en la sociedad otomana. Las contemporáneas sociedades cristiano-europeas, más rígidas y estamentales, funcionan de forma distinta. Difícilmente un humilde pescador como Dioniso Galea, futuro Uchalí, podría convertirse en el Capitán General de la armada de Felipe II. A pesar de ello, la trayectoria de Pietro Velasco pone de manifiesto un curioso mecanismo: la necesidad de conocer al enemigo, para mejor debilitarle, hace irónicamente posible que, incluso en tierras cristianas, repercutan los efectos del sistema de promoción otomano. Cautiverio, promoción del talento y rivalidades internacionales se fusionan así en el juego de la asimilación antagonista.

65 TÜRKÇELIK, Cigalazade Yusuf Sinan Pasha, pp. 14-15.

66 GRAF, Tobias (2017), "Trans-Imperial Nobility: The Case of Carlo Cigala (1556-1631)", en NORTON, Claire (ed.), Conversion and Islam in the Early Modern Mediterranean: The Lure of the Other, Routledge, Londres, pp. 9-29; y PAPPALARDO, Salvatore (2009), "Ambizione politica, commercio e diplomazia alla fine del XVI secolo: Carlo Cicala", en ZACCARIA, Claudio, ANDREOZZI, Daniele y PANARITI, Loredana (eds.), Acque, terre e spazi dei Mercanti: istituzioni, gerarchie, conflitti e pratiche dello scambio dall'età antica alla modernità, Editreg, Trieste, pp. 141-168.

67 El siglo XVII ha sido definido como la época de oro de la piratería. Cfr.: BONO, Salvatore (1993), Corsari nel Mediterraneo: cristiani e musulmani fra guerra, schiavitù e commercio, Mondadori, Milán.

68 KRSTIĆ, Tijana (2009), "Illuminated by the Light of Islam and the Glory of the Ottoman Sultanate: Self-Narratives of Conversion to Islam in the Age of Confessionalization", Comparative Studies in Society and History, $\mathrm{n}^{\circ}$ 51, pp. 35-63. El desmantelamiento de la devshirme, que a partir de la década de 1630 cae de facto en desgracia, es sólo uno de los muchos efectos de esta "confesionalización".

69 Metin Ibrahim Kunt ha acuñado esta expresión. KUNT, "Ethnic-regional (cins) solidarity", pp. 233-239. 


\section{Bibliografía}

ÁGOSTON, Gabor (2006), Guns for the Sultan: Military Power and the Weapons Industry in the Ottoman Empire, Cambridge University Press, Cambridge.

BENNASSAR, Bartolomé (1989), Los cristianos de Alá: la fascinante aventura de los renegados, Nerea, Madrid.

BONO, Salvatore (1993), Corsari nel Mediterraneo: cristiani e musulmani fra guerra, schiavitù e commercio, Mondadori, Milán.

BRAMBILLA, Elena (2000), Alle origini del sant'Uffizio. Penitenza, confessione e giustizia spirituale dal Medioevo al XVI secolo, II Mulino, Bolonia.

BRAMBILLA, Elena (2006), La giustizia intollerante. Inquisizioni e tribunali confessionali in Europa (secoli IV-XVIII), Carocci, Roma.

BUNES, Miguel Angel de (1989), La imagen de los musulmanes y del Norte de África en la España de los siglos XVI y XVII, Editorial CSIC, Madrid.

BUONFIGLIO COSTANZO, Giuseppe (1604), Prima [e secundi] parte deII'Historia siciliana, Bonifacio Ciera, Venecia.

CAPPONI, Gino (1845), Catalogo dei manoscritti posseduti dal Marchese Gino Capponi, Coi Tipi della Galileiana, Florencia.

CARALI, P. Paolo (1936), "Soggiorno di Fakhr ad-dīn al-Ma'nī in Toscana, Sicilia e Napoli e la sua visita a Malta (1613-1618)", Annali del Istituto Superiore Orientale di Napoli, $\mathrm{n}^{\circ}$ 4, pp. 15-60.

DURSTELER, Eric (2006), Venetians in Constantinople: Nation, Identity, and Coexistence in the Early Modern Mediterranean, Johns Hopkins University Press, Baltimore.

FABRIS, Antonio (1997), "Hasan 'il veneziano' tra Algeri e Costantinopoli", Quaderni di Studi Arabi, n 15 (suppl.), pp. 51-66.

FIUME, Giovanna (2010), Schiavitù mediterranee: corsari, rinnegati e santi di età moderna, Mondadori, Milán.

FLORISTÁN, José Manuel (1988), Fuentes para la política oriental de los Austrias. La documentación griega del Archivo de Simancas (1571-1621), Universidad de León, León.

FLORISTÁN, José Manuel (2016), "Relación de la empresa de Famagusta (1607)", Archivo de la Frontera, http://www.archivodelafrontera.com/ wp-content/uploads/2016/09/1607-EMPRESA-DE-FAMAGUSTA-JM-Florist\%C3\%Aln.pdf

GARCÍA-ARENAL, Mercedes (2001), Conversions islamiques: identités religieuses en islam méditerranéen, Maisonneuve et Larose, París.

GEMIGNANI, Marco (2003), "The navies of the Medici: The florentine navy 
and the navy of the sacred Military order of saint stephen, 1547-1648", en HATTENDORF, John y UNGER, Richard (eds.), War at Sea in the Middle Ages and the Renaissance, Boydell press, Woodbridge, pp. 169-185.

GIL HERRERA, Jorge y BERNABÉ, Luís F. (2014), "The Moriscos Outside Spain. Routes and Financing", en GARCÍA-ARENAL, Mercedes y WIEGERS, Gerard (eds.), The Expulsion of the Moriscos from Spain: a Mediterranean diaspora, Brill, Leiden, pp. $219-238$.

GONZÁLEZ TALAVERA, Blanca (2011), Presencia y mecenazgo español en la Florencia Medicea: de Cosme I a Fernando I. Tesis Doctoral, Universidad de Granada, Granada.

GONZALEZ-RAYMOND, Anita (1989), La Croix et le Croissant: les inquisiteurs des îles face à l'islam, 1550-1700, Centre national de la recherche scientifique, París.

GORDON, Murray (1987), L'Esclavage dans le monde arabe: Vlle-XXe siècle, Robert Laffont, París.

GRAF, Tobias (2014), "Of half-lives and double-lives: 'Renegades' in the Ottoman Empire and Their Pre-Conversion Ties, ca. 1580-1610", en FIRGES, Pascal et al. (eds.), Well-connected domains, Brill, Leiden, pp. 131-149.

GRAF, Tobias (2017), "Trans-Imperial Nobility: The Case of Carlo Cigala (1556-1631)", en NORTON, Claire (ed.), Conversion and Islam in the Early Modern Mediterranean: The Lure of the Other, Routledge, Londres, pp. 9-29.

GRAF, Tobias (2017), The Sultan's Renegades: Christian-European Converts to Islam and the Making of the Ottoman Elite, Oxford University Press, Oxford.

GÜRKAN, Emrah Safa (2014), "Money or Your Life: The Habsburg Hunt for Uluc Ali", Studia Historica: Historia Moderna, Salamanca, $n^{\circ} 36, \mathrm{pp}$. 121-145.

GÜRKAN, Emrah Safa (2016), "His Bailo's Kapudan: Conversion, Tangled Loyalties and Hasan Veneziano between Istanbul and Venice (15881591)", Journal of Ottoman Studies, $n^{\circ} 48$, pp. 277-319.

HADJIANASTASIS, Marios (2014), "Corsair tactics and lofty ideals: the 1607 Tuscan raid on Cyprus", en WALSH, Michael (ed.), Historic Famagusta: A Millennium in Words and Images, Cambridge Scholars Publishing, Cambridge, pp. 22-36.

HERRERO SÁNCHEZ, Manuel (2001), "Una república mercantil en la órbita de la Monarquía Católica (1528-1684)", en ANATRA, Bruno y MANCONI, Francesco (eds.), Sardegna, Spagna e Stati Italiani nell'Età di Carlo V, Carocci, Roma, pp. 183-200. 
HILL, George (1952), A History of Cyprus: The Ottoman Province, the British Colony, 1571-1948 (Vol. 4), Cambridge University Press, Cambridge. KOLOĞLU, Orhan (2007), "Renegades and the case Uluç/Kiliç Alì", en CANCILA, Rosaria (ed.), Mediterraneo in armi (secc. XV-XVIII), Quaderni di Mediterranea, Palermo, pp. 513-531.

KRSTIĆ, Tijana (2009), "Illuminated by the Light of Islam and the Glory of the Ottoman Sultanate: Self-Narratives of Conversion to Islam in the Age of Confessionalization", Comparative Studies in Society and History, n ${ }^{\circ} 51$, pp. 35-63. KRSTIC, Tijana (2011), Contested Conversions to Islam: narratives of Religious Change in the Early Modern Ottoman Empire, Stanford University Press, Stanford.

KUNT, Metin Ibrahim (1974), "Ethnic-Regional (cins) Solidarity in the Seventeenth-Century Ottoman Establishment", International Journal of Middle East Studies, $n^{\circ}$ 5-3, pp. 233-239.

LEWIS, Bernard (1990), Race and Slavery in the Middle East: an Historical Enquiry, Oxford University Press, Nueva York.

LUBIN, Matthew (2011), Aftermath of War: Cypriot Christians and Mediterranean Geopolitics, 1571-1625. Tesis Doctoral, The University of North Carolina at Chapel Hill, Chapel Hill.

MAS, Albert (1967), Les Turcs dans la littérature espagnole du Siècle d'Or: recherches sur l'évolution d'un thème littéraire, Centre de Recherches Hispaniques, París, Vol. 2.

MESSANA, Maria Sofia (2007), "La 'resistenza' musulmana e i 'martiri' dell'islam: moriscos, schiavi e cristiani rinnegati di fronte all'Inquisizione spagnola di Sicilia", Quaderni storici, n 42-126, pp. 743-772.

MONTICONE, Alberto (1961), Paolo Sarpi, Lettere ai gallicani. Edizione critica, saggio introduttivo e note a cura di Boris Ulianich, Franz Steiner Verlag, Wiesbaden.

MONTUORO, Domenico (2009), "I Cigala, una famiglia feudale tra Genova, Sicilia, Turchia e Calabria", Mediterranea: Richerche Storiche, $\mathrm{n}^{\circ} 6$, pp. 277-302.

OCAKAÇAN, Levent Kaya (2015), "Cigalazade Yusuf Sinan Pasha (C. 1545 1606)", Mediterranea: Richerche Storiche, Palermo, n 12, pp. 325-340.

OLDRATI, Valentina (2015), "Pedro Brea, un genovés en Constantinopla: veinte años de espionaje y contra-espionaje entre Madrid, Nápoles, Génova e Imperio otomano (1578-1596)", en SOLA CASTAÑO, Emilio y VARRIALE, Gennaro (coords.), Detrás de las apariencias: información y espionaje (siglos XVI-XVII), Universidad de Alcalá, Alcalá de Henares, pp. 153-173. 
OLDRATI, Valentina (2018), "Renegades and the Habsburg secret services in the aftermath of Lepanto: Haci Murad and the Algerian threat as a case study", Journal of Iberian and Latin American Studies, n² 24, pp. 7-26.

OLIVA, Gaetano (1907-1908), "Sinan Bassà (Scipione Cicala) celebre rinnegato del secolo XVI: Memorie storico-critiche", Archivio Storico Messinese, Mesina, $\mathrm{n}^{\circ} 8 / 9$, pp. 266-303.

PAPPALARDO, Salvatore (2009), "Ambizione politica, commercio e diplomazia alla fine del XVI secolo: Carlo Cicala", en ZACCARIA, Claudio, ANDREOZZI, Daniele y PANARITI, Loredana (eds.), Acque, terre e spazi dei Mercanti: istituzioni, gerarchie, conflitti e pratiche dello scambio dall'età antica alla modernità, Editreg, Trieste, pp. 141-168.

PRETO, Paolo (2010), I servizi segreti di Venezia: spionaggio e controspionaggio ai tempi della Serenissima, II Saggiatore, Milán.

PRETO, Paolo (2013), Venezia e i Turchi, Viella, Roma.

PROSPERI, Adriano (2011), "Firenze", en PROSPERI, Adriano, LAVENIA, Vincenzo y TEDESCHI, John (coords.). Dizionario storico dell'Inquisizione, Edizioni Normale di Pisa, Pisa, Vol. 2, pp. 605-607.

ROSTAGNO, Lucia (1983), Mi faccio turco: esperienze ed immagini dell'islam nell'Italia moderna, Istituto per l'Oriente C.A. Nallino, Roma.

ROTHMAN, E. Natalie (2012), Brokering Empire: Trans-Imperial Subjects between Venice and Istanbul, Cornell University Press, Ithaca.

SCARAFFIA, Lucetta (1993), Rinnegati: per una storia dell'identità occidentale, Laterza, Roma.

SIEBENHÜNER, Kim (2008), "Conversion, mobility and the Roman Inquisition in Italy Around 1600", Past \& Present, , n² 200-1, pp. 5-35.

SOLA, Emilio (2005), Los que van y los que vienen: información y fronteras en el Mediterráneo clásico del siglo XVI, Universidad de Alcalá, Alcalá de Henares.

SOLA, Emilio (2007), La Conjura de Campanela, Turpin, Madrid.

SOLA, Emilio (2011), Uchali: el calabrés tiñoso o el mito del corsario muladi en la frontera, Ediciones Bellaterra, Barcelona.

SOLA, Emilio y DE LA PEÑA, José (1995), Cervantes y la Berbería: Cervantes, mundo turco-berberisco y servicios secretos en la época de Felipe II, Fondo de Cultura Económica, Madrid.

TÜRKÇELIK, Evrim (2013), "El renegado 'El Cigala' y la política mediterránea", Desperta Ferro. Historia Moderna, n 6, pp. 40-43. 
TÜRKÇELIK, Evrim (2012), Cigalazade Yusuf Sinan Pasha y el Mediterráneo entre 1591-1606. Tesis Doctoral, Universidad Autónoma de Madrid, Madrid.

VARRIALE, Gennaro (2014), Arrivano li Turchi: guerra navale e spionaggio nel Mediterraneo (1532-1582), Città del Silenzio, Novi Ligure.

VITALI, Francesco (2017), I nunzi pontifici nella Firenze di Ferdinando I (1587-1609), Nuova Cultura, Roma. 